\title{
CONSEIL EN COMPTABILITÉ ADMINISTRATIVE : UNE NOUVELLE FAÇON DE GÉRER UNE ENTREPRISE VIA LA TECHNOLOGIE
}

\section{ARTICLE ORIGINAL}

SUMAR, Ramiro Rodrigues ${ }^{1}$

SUMAR, Ramiro Rodrigues. Conseil en comptabilité administrative : une nouvelle façon de gérer une entreprise via la technologie. Revista Científica Multidisciplinar Núcleo do Conhecimento. An 06, Ed. 07, Vol. 12, p. 91 à 104. Juillet 2021. ISSN : 2448-0959, Lien d'accès: https://www.nucleodoconhecimento.com.br/administration-des-affaires/via-la-

technologie, DOI: 10.32749/nucleodoconhecimento.com.br/administration-desaffaires/via-la-technologie

\section{RÉSUMÉ}

Objectif : Décrire l'impact des obstacles et des potentialités que la technologie a apportés aux conseils en comptabilité. Question : Comment le conseil en comptabilité administrative peut-il aider une entreprise à utiliser des technologies ? Méthodologie : L'étude est une revue bibliographique. Une recherche a été effectuée avec les descripteurs : technologie; l'innovation; comptabilité; comptabilité sur la plateforme de données de Spell (Scientific Periodicals Electronic Library) en juin 2021. La recherche a abouti à 10 articles. Résultats: les résultats apportés comme obstacles: L'avancée technologique croissante, s'adapter à l'utilisation de ces nouvelles technologies, Centralité du rôle du comptable dans les modèles traditionnels, Manque de connaissances logicielles,Manque de main-d'œuvre spécialisée, dysfonctionnement de la connexion, Main-d'œuvre qualifiée. Et comme potentialités: Utilisation de différentes technologies numériques, Agilité et gain de

\footnotetext{
${ }^{1}$ Maîtrise en contrôle, spécialiste en gestion de la technologie, administration stratégique, sécurité de I'information et diplômé en sciences comptables.
}

RC: 93886

Disponível em: https://www.nucleodoconhecimento.com.br/administration-des- 
temps, Commodité, relation inséparable entre la technologie et la comptabilité consultative favorisant un support agile et de qualité à ses différents utilisateurs, Satisfaction du client, Rapidité et flexibilité et Réduction des coûts. Conclusions: II est entendu que les technologies de l'information influencent positivement l'exercice de la comptabilité consultative, bien qu'en plus des avantages a ses inconvénients. II convient de mentionner que les technologies permettent de contrôler les informations comptables pour l'entrée et la sortie des matériaux et des intrants, ainsi que leur origine et leur destination finale, en évitant les pertes inutiles, y compris les écarts, l'agilité, la flexibilité et la sécurité.

Mots-clés : Conseil, Innovation, Moyens Technologiques, Sécurité de l'Information.

\section{INTRODUCTION}

Le lien entre la comptabilité et les technologies s'est perpétué pendant des décennies, car, en plus d'être scientifique, la comptabilité est l'un des systèmes d'information utilisés dans la gestion d'entreprise plus ancienne, ce qui rend la comptabilité du passé et la comptabilité du présent différentes sont les façons dont elles conduisent à la gestion d'entreprise (FERREIRA et al., 2017).

La technologie a progressé au fil des ans, innovant de sorte que le besoin de l'être humain est satisfait. Par conséquent, étant un outil illimité, l'évolution des technologies combinée à la comptabilité apporte des contributions pertinentes à la croissance des organisations (SILVA et al., 2020).

L'utilisation continue des réseaux informatiques et la combinaison de divers instruments comptables favorisent le stockage, l'enregistrement, le traitement et facilitent la réalisation du croisement de l'information de plus en plus rapidement, ce qui permet une prise de décision plus sûre au sein de l'organisation (MARION; RIBEIRO, 2017; DE ANDRADE SIMÕES et al., 2021).

RC: 93886

Disponível em: https://www.nucleodoconhecimento.com.br/administration-des- 
À l'heure actuelle, la recherche d'informations a été utile et en même temps pertinente pour la croissance de l'organisation. Le transfert de l'information au savoir, ainsi que l'application opportune du savoir dans la pratique et la distribution de ces actes pour le développement d'une entreprise, sont des potentialités de la technologie en faveur de la comptabilité consultative pour l'entrepreneur.

Cependant, de nombreux comptables n'utilisent pas cet outil comme un moyen de gagner de l'espace, du temps et de fidéliser leurs clients, indiquant clairement que dans certaines situations, les technologies pour les compteurs ne sont pas utilisées. Dans ce placement, identifiant la problèmeatisation de l'étude en question, il y a la question qui a guidé le thème de cette étude: Comment le conseil en comptabilité administrative peut-il aider une entreprise utilisant des technologies?

Le marché du travail actuel motive souvent les gens à se reformuler dans leurs activités professionnelles, parce que le système d'enseignement universitaire de base et les nouvelles connaissances deviennent encore peu nombreux compte tenu de la pratique que l'étudiant effectuera après l'obtention de son diplôme.

Selon Villa et al (2012, p. 107), " les comptables traditionnels sont d'avis que les entrepreneurs ne recherchent pas les connaissances nécessaires pour gérer leur entreprise, c'est-à-dire qu'ils ne prennent pas de cours de perfectionnement ou de formation en administration ". Pour paraphraser cette citation de l'auteur, indique que l'entrepreneur n'a que l'intérêt de répondre aux exigences des impôts, oubliant ainsi le marché concurrentiel, qui fait perdre de l'espace à l'entreprise et ne se développe pas dans sa qualité.

Dans ce placement, l'importance du compteur consultatif est observée. Ce travail s'ajoute à la justification du chercheur de comprendre pourquoi de nombreux comptables n'utilisent pas la comptabilité consultative pour travailler avec leurs clients, car cet outil d'aide, en particulier en période de pandémie du nouveau

RC: 93886

Disponível em: https://www.nucleodoconhecimento.com.br/administration-des- 
coronavirus, exerce une influence afin que l'entreprise ne risque pas d'avoir un engagement dans ses revenus sur le marché concurrentiel.

Par conséquent, le sujet devient pertinent, car avec l'avènement des technologies, la comptabilité des entreprises a pris une direction différente de la comptabilité il y a des décennies et avec cela, le comptable a maintenant la responsabilité et l'engagement de faire de sa profession identifiée par la société et les entrepreneurs comme une profession qui progresse pour s'adapter aux moyens technologiques afin d'assurer plus de sécurité et de fiabilité aux organisations.

Par conséquent, ce travail a pour objectif général: décrire l'impact des obstacles et des potentialités que la technologie a apportés au conseil en comptabilité.

\section{PROCESSUS MÉTHODOLOGIQUE}

Cette étude est une revue bibliographique systématique de nature qualitative. Selon Sampaio et Mancini (2007), ce type d'étude est réalisé par la recherche d'articles pertinents au thème étudié, sur la base de la synthèse de sujets pertinents combinée à des méthodes d'investigation systématisées, d'analyse critique, de résumé des informations choisies et de problèmes importants qui nécessitent des études supplémentaires.

Pour mener à bien la recherche, les étapes suivantes recommandées par Sampaio et Mancini (2007) : Formulation de la question-guide de l'étude ont été suivies; Lieu des études; Évaluation critique des études; Collecte de données; Analyse des données; Aspects éthiques.

De la construction d'une question de guide, certaines hypothèses se dégagent qui guident et/ou délimitent cette étude, permettant ainsi de mettre en évidence l'applicabilité des technologies dans l'univers de la consultation des nombres et des valeurs auxquels le comptable est inséré. La question qui a guidé le sujet était la

RC: 93886

Disponível em: https://www.nucleodoconhecimento.com.br/administration-des- 
suivante : comment le conseil en comptabilité administrative peut-il aider une entreprise à utiliser des technologies?

Le matériel a été sélectionné dans les bases de données: Spell (Scientific Periodicals Eletronic Library). L'échantillon de l'étude se composait d'articles (recherche originale; articles de synthèse; monographies, thèses et mémoires). Les articles en portugais et en anglais ont été utilisés comme critères d'inclusion, de 2016 à 2020, qui sont disponibles dans leur intégralité et gratuitement. Tous les articles qui étaient encadrés dans ces critères, qui, dans leur domaine de connaissances, couvraient la comptabilité et avaient comme titre ou sujet abstrait pertinent pour l'utilisation des technologies, ont été encadrés pour la recherche. D'autres articles qui n'étaient pas inclus ou qui n'étaient pas reproduits ont été exclus. Les mots-clés utilisés pour rechercher ces articles dans Spell étaient: technologie; l'innovation; comptabilité; comptabilité consultative.

L'évaluation critique des productions sélectionnées et incluses dans l'étude a donné lieu à des interprétations et à une synthèse des résultats, identifiant les informations scientifiques présentes dans chaque étude et les facteurs pertinents pour l'étude en question suggérant des lignes directrices pour la recherche future. Pour valider l'étude, le chercheur indique clairement quelles lacunes ont été trouvées dans l'étude et quelles voies futures d'autres chercheurs peuvent adopter dans leur recherche scientifique. Les principales informations ont été organisées en tableaux, indiquant clairement les informations pertinentes pour les résultats des articles, comfacant les sujets avec la littérature pertinente pour le sujet.

Les données ont été recueillies en juin 2021. La collecte a été effectuée, y compris les critères d'inclusion et d'exclusion des articles. Soulignant également que la recherche vise également, sur la base de l'analyse effectuée, à rendre compte de la comptabilité consultative et de l'utilisation des technologies. Deux recherches ont été effectuées entrecoupant les mots-clés.

$\mathrm{RC}: 93886$

Disponível em: https://www.nucleodoconhecimento.com.br/administration-des- 
Les données trouvées dans la littérature publiée se sont produites selon la proposition d'Ercole ; Melo et Alcoforado (2014), dans lesquels l'évaluateur devrait chercher à évaluer les résultats de façon impartiale, en cherchant des explications pour les résultats différents ou contradictoires dans les différentes études.

\section{ANALYSE DES RÉSULTATS ET DISCUSSIONS}

Les recherches ont abouti à un total de 343 résultats avec les mots-clés. Tous les titres et résumés ont été lus et les articles qui pouvaient répondre aux objectifs de l'étude en question ont été supprimés, ce qui a donné lieu à 10 études.

Ainsi, nous présentons une image avec le titre de l'article, les auteurs, l'année de publication (Référence), l'objectif de l'étude et les résultats trouvés.

Tableau 1 - Sélection d'articles

\begin{tabular}{|c|c|c|}
\hline $\begin{array}{l}\text { Titre/Référence de } \\
\text { l'article }\end{array}$ & buts & Résultats \\
\hline $\begin{array}{l}\text { No caminho da } \\
\text { inovação: análise das } \\
\text { capacidades de } \\
\text { inovação de } \\
\text { empresas contábeis } \\
\text { diante } \\
\text { tecnologias digitais }\end{array}$ & $\begin{array}{l}\text { Examiner la capacité } \\
\text { d'innovation des bureaux } \\
\text { comptables sur le marché } \\
\text { brésilien, dans l'utilisation } \\
\text { des technologies } \\
\text { numériques, sur la base } \\
\text { des capacités de } \\
\text { développement, } \\
\text { d'exploitation, de gestion et } \\
\text { de transaction } \\
\text { technologiques. }\end{array}$ & $\begin{array}{l}\text { La comptabilité commence sur } \\
\text { la voie traditionnelle de } \\
\text { l'innovation numérique, } \\
\text { démontrant la qualité et la } \\
\text { valeur que les solutions } \\
\text { technologiques peuvent } \\
\text { générer lorsqu'elles sont } \\
\text { exploitées dans les affaires et } \\
\text { en particulier dans les } \\
\text { processus.Cette augmentation } \\
\text { de la technologie entraîne des } \\
\text { changements dans les } \\
\text { modèles d'affaires }\end{array}$ \\
\hline
\end{tabular}

RC: 93886

Disponível em: https://www.nucleodoconhecimento.com.br/administration-des- 


\begin{tabular}{|c|c|c|}
\hline & & \\
\hline $\begin{array}{l}\text { Indústria } 4.0 \text { e } \\
\text { avanços tecnológicos } \\
\text { da área contábil: } \\
\text { perfil, percepções e } \\
\text { expectativas dos } \\
\text { profissionais } \\
\text { Xavier ; Carraro et } \\
\text { Rodrigues (2020). }\end{array}$ & $\begin{array}{l}\text { Analyser le profil du } \\
\text { professionnel de la } \\
\text { comptabilité face aux } \\
\text { avancées technologiques, } \\
\text { en visant à explorer } \\
\text { comment il se comporte par } \\
\text { rapport aux nouvelles } \\
\text { tendances. }\end{array}$ & $\begin{array}{l}\text { La plupart des professionnels } \\
\text { sont intéressés à s'adapter } \\
\text { aux nouvelles technologies } \\
\text { pour répondre aux exigences } \\
\text { de la fonction. Cependant, il y } \\
\text { a eu quelques résistances aux } \\
\text { changements, en particulier } \\
\text { chez les professionnels de } \\
\text { moins de } 35 \text { ans et dans le } \\
\text { domaine des entreprises. }\end{array}$ \\
\hline $\begin{array}{l}\text { Características dos } \\
\text { Diferentes } \\
\text { de Modelos } \\
\text { Contábeis } \\
\text { Relação às Áreas da } \\
\text { Contabilidade }\end{array}$ & $\begin{array}{l}\text { Identifier la valeur fournie } \\
\text { au client des services } \\
\text { comptables, à travers } \\
\text { l'organisation structurelle et } \\
\text { les caractéristiques des } \\
\text { modèles d'affaires } \\
\text { traditionnels, par rapport à } \\
\text { leurs domaines d'activité } \\
\text { respectifs en comptabilité. }\end{array}$ & $\begin{array}{l}\text { Le domaine de la comptabilité } \\
\text { axé sur les systèmes } \\
\text { d'information a ses livraisons } \\
\text { basées sur le conseil en } \\
\text { traitement des données, en } \\
\text { technologie de l'information et } \\
\text { en systèmes d'exploitation. }\end{array}$ \\
\hline $\begin{array}{l}\text { As Contribuições da } \\
\text { Contabilidade } \\
\text { Consultiva: Um } \\
\text { Estudo de Caso/The } \\
\text { Contributions of } \\
\text { Advisory Accounting: } \\
\text { A Case Study }\end{array}$ & $\begin{array}{l}\text { Vérifiez les contributions } \\
\text { favorisées par l'avancement } \\
\text { de la technologie en } \\
\text { comptabilité ainsi qu'en } \\
\text { comptabilité consultative. }\end{array}$ & $\begin{array}{l}\text { Il a été constaté que l' } \\
\text { perçoivent l'importance de la } \\
\text { comptabilité consultative dans } \\
\text { le scénario actuel ainsi que le } \\
\text { rôle essentiel de la } \\
\text { technologie dans la profession } \\
\text { comptable. Les défis abordés } \\
\text { par les personnes interrogées } \\
\text { comprennent l'adaptation à }\end{array}$ \\
\hline
\end{tabular}

RC: 93886

Disponível em: https://www.nucleodoconhecimento.com.br/administration-des- 
(2020).

\section{Impact of information Déterminer l'impact} technology

on technologies

accounting line of l'information sur la ligne works

Asuquo; Dan et comptable des travaux dans le système mondial.

Effiong (2020).

The impact of
information and
communication
technology (ICT) in
the accounting
system: advantages,
applications, and
challenges

Al-Shafeay; Al_Dujaili

et Al-Wattar (2020). l'utilisation de nouveaux outils technologiques, la nécessité de définir des stratégies pour mieux servir le client et la nécessité de connaître la gestion et les affaires de chaque client.

des Les résultats de la recherche de ont montré que les technologies de l'information ont une influence considérable sur le secteur de travail comptable.

The Impact of Déterminer si la technologie La recherche a montré que la Information a eu un effet (positif ou rapidité et la flexibilité sont Technology on The négatif) sur les applications l'effet le plus technologique Quality of Accounting comptables et si la sur les applications

RC: 93886

Disponível em: https://www.nucleodoconhecimento.com.br/administration-des- 


\begin{tabular}{|c|c|c|}
\hline $\begin{array}{l}\text { Information } \quad \text { (SFAC } \\
\text { NO 8, 2010) }\end{array}$ & $\begin{array}{l}\text { technologie de l'information } \\
\text { en comptabilité a un effet } \\
\text { sur la qualité des rapports } \\
\text { financiers en Arabie } \\
\text { saoudite. }\end{array}$ & $\begin{array}{l}\text { comptables avec des } \\
\text { caractéristiques améliorées et } \\
\text { fiables. }\end{array}$ \\
\hline $\begin{array}{l}\text { A Consultoria } \\
\text { Contábil como } \\
\text { Oportunidade de } \\
\text { Negócios } \\
\text { Profissionais - Um } \\
\text { Estudo Documental }\end{array}$ & $\begin{array}{l}\text { Mener une étude } \\
\text { documentaire et } \\
\text { bibliométrique sur le conseil } \\
\text { en comptabilité au cours de } \\
\text { la période de } 2006 \text { à } 2016 \text {. }\end{array}$ & $\begin{array}{l}\text { Le cabinet de conseil en } \\
\text { comptabilité présente un } \\
\text { facteur de rendement financier } \\
\text { qualitatif pour les entreprises, } \\
\text { augmentant ainsi l'avantage } \\
\text { concurrentiel pour elles. }\end{array}$ \\
\hline
\end{tabular}

Almeida et al. (2018).

Consultoria

Organizacional como um Instrumento de Inovação em Arranjos Produtivos Locais

Silva; Ribeiro e Silva (2018).

\section{Analyser comment les} pratiques de consultation organisationnelle peuvent déclencher l'innovation dans les arrangements productifs locaux (APL).

Planejamento

Estratégico:

Diagnóstico

Proposta Intervenção por Meio de Consultoria

Gafuri; Rojo et Miura (2017).

\section{Diagnostiquer et présenter} des propositions

d'intervention par le biais de de conseils qui contribuent à la mise en forme d'une planification stratégique pour une entreprise active dans le domaine des chaussures et accessoires
Les consultants ont apporté des innovations à la plupart des entreprises, étant $89 \%$ dans les processus, $77 \%$ dans la gestion d'entreprise, 74\% dans les produits et $40 \%$ dans le marketing.

\section{Grâce à une approche de} conseil externe, une vision externe de l'organisation, il est indispensable d'adapter les techniques et les outils au type d'entreprise analysé, ainsi qu'à l'activité exercée, à la taille de l'entreprise et au volume des négociations.

RC: 93886

Disponível em: https://www.nucleodoconhecimento.com.br/administration-des- 
pour femmes de boutique.

Source : Paternité propre

Les articles sélectionnés ont identifié les obstacles à l'utilisation des technologies par le comptoir. Le tableau 2 présente une synthèse de ces difficultés à partir de la référence de l'article sélectionné.

Tableau 2 - Obstacles rencontrés

\section{Titre de l'article, Référence}

No caminho da inovação: análise das capacidades de inovação de empresas contábeis diante das tecnologias digitais Indústria 4.0 e avanços tecnológicos da área contábil: perfil, percepções e technologies expectativas dos profissionais

Características dos Diferentes Le rôle central du comptable dans les Modelos de Negócios Contábeis em modèles d'affaires comptables traditionnels, Relação às Áreas da Contabilidade et les ressources technologiques sont toujours à l'appui dans ce scénario.

As Contribuições da Contabilidade S'adapter à l'utilisation des nouveaux outils Consultiva: Um Estudo de Caso/The technologiques

Contributions of Advisory Accounting:

A Case Study

Impact of information technology on Conservez les goûts détectables de votre accounting line of works passé enracinés dans le caractère et l'intégrité

The impact of information and Gestionnaires de projet inexpérimentés;

RC: 93886

Disponível em: https://www.nucleodoconhecimento.com.br/administration-des- 
communication technology (ICT) in Manque de connaissances en logiciels; the accounting system: advantages, Contrats incomplets; Manque de mainapplications, and challenges

The Impact of Information Moyens de faire face à des problèmes Technology on The Quality of électriques, un dysfonctionnement de la Accounting Information (SFAC NO 8, connexion Internet et des virus informatiques. 2010)

A Consultoria Contábil como Durée plus longue et allocation de Oportunidade de Negócios ressources et de coûts d'exécution plus Profissionais - Um Estudo importants.

Documental

Consultoria Organizacional como um Main-d'œuvre qualifiée. Instrumento de Inovação em Arranjos

Produtivos Locais

Planejamento Estratégico: Manque de connaissances en logiciels.

Diagnóstico e Proposta de Intervenção por Meio de Consultoria

Source : Paternité propre

On observe qu'il y a plusieurs obstacles mis en évidence dans les articles en ce qui concerne l'utilisation des technologies pour le conseil en comptabilité. Ces obstacles proviennent principalement des difficultés de nombreux professionnels qui ne s'adaptent pas à l'utilisation des technologies dans leur routine, ou parce que le professionnel n'est pas correctement qualifié ou qualifié, ou par crainte des changements technologiques.

Schiavi et al (2020) soulignent que les technologies doivent être considérées comme un support pour viser de bons résultats, en particulier dans les cabinets de conseil, cependant, le comptable doit être conscient des avancées technologiques qui ne

RC: 93886

Disponível em: https://www.nucleodoconhecimento.com.br/administration-des- 
sont pas lentes, ce qui permet au professionnel d'avoir peur d'utiliser la technologie comme un support ciblé pour le conseil.

Xavier ; Carraro et Rodrigues (2020) mentionnent que la comptabilité consultative doit être dirigée par des moyens technologiques, car elle favorise plus de sécurité, de précision et de fiabilité dans les données trouvées, cependant, pour que cela se produise, il appartient au comptable d'être conscient des changements dans les avancées technologiques et de s'adapter à l'ensemble du système, en apportant au client des technologies avancées et de première ligne.

Schiavi et Behr (2020) et Angelim et Barreto (2020) signalent qu'une partie des professionnels de la comptabilité ont toujours leur domaine dans la tradition de la science comptable, ne permettant pas la présence totale de technologies de la meilleure qualité dans les services de conseil en comptabilité, ce qui reflète un retard important dans la découverte de la prise de décision.

Il est nécessaire pour le professionnel d'être conscient des nouvelles tendances technologiques pour l'amélioration de l'entreprise, un comptable formé à l'utilisation des technologies tend à réduire le temps pour atteindre l'équilibre de l'entreprise, ainsi que de réussir à passer la fiabilité dans les données collectées.

Selon Asuquo; Dan et Effiong (2020) comptabilité consultative apporte la possibilité de se développer dans tous les secteurs de la comptabilité, que ce soit de manière managériale ou financière, ce qui détermine le travail de conseil est la raison pour laquelle le conseil sera travaillé, cependant, l'enracinement de la comptabilité traditionnelle, crée un énorme obstacle entre le comptable et les technologies, où ce professionnel se sent encore plus en sécurité en présence du client dans son bureau.

Le manque de connaissance des technologies et de leurs avantages imprègne encore la vie de nombreux professionnels. Le manque de formation professionnelle,

RC: 93886

Disponível em: https://www.nucleodoconhecimento.com.br/administration-des- 
le manque de connaissances sur les programmes, la main-d'œuvre qualifiée, entre autres, permettent aux technologies de ne pas entrer dans le conseil en comptabilité et donc de favoriser rapidement, en toute sécurité et efficacement, l'analyse des données et, par conséquent, la prise de décision (AL-SHAFEAY; AL_DUJAILI; ALWATTAR, 2020; ALMEIDA et al., 2018; SILVA; RIBEIRO; SILVA, 2018; GAFURI, ROJO; MIURA, 2017).

Cependant, les obstacles ne concernent pas seulement les difficultés des comptables dans l'utilisation des technologies, le dysfonctionnement des programmes, la lenteur d'Internet, les virus dans l'ordinateur peuvent influencer l'inaptituance du professionnel au support des technologies pour aider à la comptabilité consultative (AL NATOUR, 2020).

L'utilisation du logiciel et du matériel nécessite un dévouement intense du professionnel pour pouvoir savoir comment utiliser les outils et avec cela les méthodes actuelles qui peuvent apporter des coûts inférieurs et de plus grands avantages à l'entreprise. Compte tenu de ces obstacles dans leur complexité de l'environnement technologique, il identifie les potentialités que les technologies peuvent apporter au conseil en comptabilité.

Tableau 3 - Possibilités de consultation comptable et d'utilisation des technologies

\section{Titre de l'article, Référence}

No caminho da inovação: análise das capacidades de inovação de empresas contábeis diante das tecnologias digitais Indústria 4.0 e avanços tecnológicos da área contábil: perfil, percepções e expectativas dos profissionais

\section{Potentiel}

Utilisation de différentes technologies numériques; agir entièrement en ligne pour apporter plus d'accessibilité et d'agilité dans la prestation de services comptables Agilité et gain de temps. Commodité.

RC: 93886

Disponível em: https://www.nucleodoconhecimento.com.br/administration-des- 
Características dos Diferentes L'utilisation de l'utilisation des moyens Modelos de Negócios Contábeis em technologiques de l'utilisateur tels que les eRelação às Áreas da Contabilidade mails, le téléphone, entre autres pour effectuer des conseils comptables.

As Contribuições da Contabilidade Relation inséparable entre technologie et Consultiva: Um Estudo de Caso/The comptabilité consultative favorisant un Contributions of Advisory Accounting: support agile et de qualité auprès de ses A Case Study

Impact of information technology on différents utilisateurs.

accounting line of works

Satisfaire vos publics internes et externes en leur offrant des services de qualité et de performance sur une base continue et cohérente.

The impact of information and Satisfaction de la clientèle; Agilité; Fiabilité. communication technology (ICT) in the accounting system: advantages, applications, and challenges

The Impact of Information Rapidité et flexibilité Technology on The Quality of Accounting Information (SFAC NO 8, 2010)
A Consultoria Contábil como
Risque des résultats attendus des travaux à Oportunidade de Negócios effectuer
Profissionais - Um Estudo
Documental
Consultoria Organizacional como um Instrumento de Inovação em Arranjos Produtivos Locais
Nouvelles méthodes d'organisation du travail; Nouvelles techniques de gestion des connaissances; Changements importants dans les relations avec d'autres entreprises
Planejamento Estratégico: Réduction des coûts, contrôle de gestion

RC: 93886

Disponível em: https://www.nucleodoconhecimento.com.br/administration-des- 
Diagnóstico e Proposta de avec déploiement pour les calendriers Intervenção por Meio de Consultoria d'achat.

Source : Paternité propre

Il est évident que la nécessité d'approfondir de nouveaux concepts et de nouvelles connaissances est d'une importance capitale pour les professionnels de la comptabilité, pour cela, le potentiel des technologies de conseil en comptabilité est observé. La comptabilité est considérée comme un outil fondamental pour soutenir la croissance de l'entreprise, cependant, le manque de connaissance des technologies par le comptable rend cette connaissance acquise identifiée dans la traditionalité des rôles.

Schiavi et al. (2020) mentionnent plusieurs potentialités que les technologies peuvent favoriser le conseil en comptabilité, la citation est: Utilisation de différentes technologies numériques; agir entièrement en ligne pour apporter plus d'accessibilité et d'agilité dans la prestation de services comptables. Ces positions des auteurs identifient l'importance de la comptabilité et des technologies qui se côtoient toujours, ce qui favorise la fidélité du comptable à ses clients.

Xavier ; Carraro et Rodrigues (2020) corroborent avec les auteurs ci-dessus sur les potentialités des technologies de conseil en comptabilité et renforcent qu'en plus de ces placements, on peut également mentionner l'agilité, le gain de temps et la commodité de l'entreprise et du comptable.

Pour Schiavi et Behr (2020), l'utilisation de technologies pour effectuer des conseils comptables n'est pas seulement liée à des questions de programmes informatiques, de téléphone, de réseaux sociaux, de courriels, entre autres technologies, peut aider le comptable traditionnel à effectuer ses consultations sans nécessairement avoir à travailler avec des programmes.

RC: 93886

Disponível em: https://www.nucleodoconhecimento.com.br/administration-des- 
Selon Angelim et Barreto (2020), les technologies ont déjà leur participation à la comptabilité consultative, cependant, la présence de programmes facilitants pour la réalisation du conseil en comptabilité a conduit à ce qu'on appelle la technologie d'innovation, apportant agilité et plus de fiabilité dans les données présentées.

Selon Asuquo; Dan et Effiong (2020) et Al-Shafeay; Al Dujaili et Al-Wattar (2020), la comptabilité consultative vise à fournir les informations les plus fiables sur l'entreprise et les résultats qu'elle obtient, la satisfaction du client en apportant des services de qualité et de performance est ce qui démontre la différence de la présence de la technologie la plus avancée pour le comptable.

Al Natour (2020) que les technologies sont rapides et flexibles, deux conditions essentielles pour le travail du comptable qui dans son temps de conseil devient un ennemi pour la prise de décision, car le marché concurrentiel est de plus en plus exigeant vis-à-vis des entreprises.

Selon Almeida et al. (2018) les tendances à l'augmentation du bénéfice d'une entreprise ont une incidence directe sur le comptable, ce qui renforce les connaissances comptables en matière de prise de risque. Les technologies agissent afin d'assurer moins de risques pour le comptable et une meilleure rentabilité à l'entreprise, ce qui permet au client et au propriétaire de l'entreprise un service de qualité et dans un temps agile.

Silva; Ribeiro e Silva (2018) e Gafuri; Rojo et Miura (2017) soulignent qu'en plus de fournir une analyse précise et sûre, les technologies fournissent également des méthodes d'organisation du travail, de gestion des connaissances, une meilleure relation avec d'autres entreprises, un contrôle des coûts, une amélioration de la prise de décision, entre autres potentialités visant à une plus grande productivité avec des coûts inférieurs par rapport aux techniques traditionnelles et manuelles.

RC: 93886

Disponível em: https://www.nucleodoconhecimento.com.br/administration-des- 
Il est perçu que les technologies combinées à la comptabilité consultative ont plusieurs potentialités qui permettent à la comptabilité d'avoir sa prise de décision afin que l'entreprise n'ait pas de problèmes financiers ou ne quitte pas le marché concurrentiel, agissant en plus du problème mis en évidence.

\section{CONSIDÉRATIONS FINALES}

Les obstacles à l'utilisation des technologies en comptabilité consultative restent préoccupants, considérant que le professionnel de la comptabilité devrait avoir comme aide à la technologie pour améliorer son service et que la non-acceptation de l'utilisation des technologies peut entraîner la disqualification et le discrédit de ce professionnel de ses fonctions.

Le professionnel de la comptabilité doit garder à l'esprit les pratiques durables pour pouvoir générer plus d'avantages financiers et opérationnels pour les entreprises, étant donné qu'en plus du coût élevé d'acquisition et de maintenance de l'ensemble du processus d'installation technologique, il y a des dépenses en matière de qualification et de formation professionnelle.

La demande croissante de programmes informatiques influence directement la nonacceptation de nombreux professionnels, cela peut être considéré de manière désavantageuse pour la comptabilité, car il n'existe pas de système unique pour effectuer les activités nécessaires dont la comptabilité a besoin.

Mais le potentiel que les progrès technologiques apportent au conseil en comptabilité est immense, et ces potentialités peuvent générer des impacts positifs concernant l'acceptation de l'utilisation des technologies pour atteindre les objectifs en comptabilité. Dans ce contexte, les technologies facilitent la vue d'ensemble du compteur, dans lequel la perception d'une marge d'erreur plus faible et l'accès à toutes les données d'intérêt qui peuvent être compilées et / ou facturées, trouvent dans la technologie un allié pour effectuer un service d'excellence.

$\mathrm{RC}: 93886$

Disponível em: https://www.nucleodoconhecimento.com.br/administration-des- 
On considère donc que l'étude atteint l'objectif qui lui est indiqué et qu'elle renvoie comme une lacune à la recherche menée sur la base du conseil comptable en général, ce qui ouvre la voie à l'idéalisation de nouvelles recherches en étendant ce thème à une forme plus restreinte telle que le conseil en comptabilité financière ou même en comptabilité de gestion.

\section{RÉFÉRENCES}

ALMEIDA, Leonardo Soares Francisco de et al. A Consultoria Contábil como Oportunidade de Negócios Profissionais-um Estudo Documental. Pensar Contábil, v. 20, n. $72,2018$.

AL NATOUR, Jihad Rebhee Abdel Qader et al. The Impact of Information Technology on The Quality of Accounting Information (SFAC NO 8, 2010). Turkish Journal of Computer and Mathematics Education (TURCOMAT), v. 12, n. 13, p. 885-903, 2020.

AL-SHAFEAY, Karrar Mohammed; AL_DUJAILI, Mohammed Jawad; AL-WATTAR, Yasir Mohammed Ali. The impact of Information and Communication Technology (ICT) In the accounting system: advantages, applications, and challenges. PalArch's Journal of Archaeology of Egypt/Egyptology, v. 17, n. 6, p. 15814-15825, 2020.

ANGELIM, Vitória Ribeiro; BARRETO, Tayssa Vieira. As Contribuições da Contabilidade Consultiva: Um Estudo de Caso/The Contributions of Advisory Accounting: A Case Study. ID on line Revista de Psicologia, v. 14, n. 52, p. 317$331,2020$.

ASUQUO, Akabom I.; DAN, Nicholas O.; EFFIONG, Glory T. Impact of information technology on accounting line of works. International Journal of Recent Technology and Engineering, v. 9, n. 2, p. 1572-1577, 2020.

$\mathrm{RC}: 93886$

Disponível em: https://www.nucleodoconhecimento.com.br/administration-des- 
DE ANDRADE SIMÕES, Maervelym Pâmella et al. Benefícios do uso da tecnologia Blockchain como instrumento para a auditoria contábil. Revista ambiente contábilUniversidade Federal do Rio Grande do Norte-ISSN 2176-9036, v. 13, n. 1, p. 3953, 2021.

ERCOLE, Flávia Falci; MELO, Laís Samara de; ALCOFORADO, Carla Lúcia Goulart Constant. Revisão integrativa versus revisão sistemática. Revista Mineira de Enfermagem, v. 18, n. 1, p. 9-12, 2014.

FERREIRA, Tiago Janes et al. Automação contábil: tecnologia em contabilidade sob a ótica da teoria institucional. XI Congresso ANPCONT. 3 a 6 de junho de 2017. Belo Horizonte, Minas Gerais.

GAFURI, Raquel Adriana Pin; ROJO, Cláudio Antonio; MIURA, Marcio Nakayama. Planejamento estratégico: diagnóstico e proposta de intervenção por meio de consultoria. Revista Capital Científico-Eletrônica (RCCe)-ISSN 2177-4153, v. 15, n. 1, p. 139-155, 2017.

MARION, JOSE CARLOS; RIBEIRO, OSNI MOURA. Introdução à contabilidade gerencial. Saraiva Educação SA, 2017. Disponível em: https://books.google.com.br/books?hl=pt-

$B R \& \mid r=\& i d=k D 1 n D w A A Q B A J \& o i=f n d \& p g=P T 5 \& d q=I n t r o d u \% C 3 \% A 7 \% C 3 \% A 30+\% C$ $3 \% \mathrm{~A} 0+$ contabilidade+gerencial.+\&ots=JjrSnq2mD0\&sig=vcUiQXgi1UkNhLanfgWU67 QVgas\#v=onepage \&q=Introdu\%C3\%A7\%C3\%A30\%20\%C3\%A0\%20contabilidade\% 20gerencial.\&f=false

SAMPAIO, Rosana Ferreira; MANCINI, Marisa Cotta. Estudos de revisão sistemática: um guia para síntese criteriosa da evidência científica. Brazilian Journal of Physical Therapy, v. 11, p. 83-89, 2007.

RC: 93886

Disponível em: https://www.nucleodoconhecimento.com.br/administration-des- 
SCHIAVI, Giovana Sordi; BEHR, Ariel. Características dos diferentes modelos de negócios contábeis em relação às áreas da Contabilidade. REUNIR Revista de Administração Contabilidade e Sustentabilidade, v. 10, n. 3, p. 47-59, 2020.

SCHIAVI, Giovana Sordi et al. No caminho da inovação: análise das capacidades de inovação de empresas contábeis diante das tecnologias digitais. Revista Brasileira de Gestão de Negócios, v. 22, p. 381-405, 2020.

SILVA, Gustavo Oliveira et al. O impacto da tecnologia na profissão contábil sob perspectivas de pessoas com formação e/ou experiência profissional na área. Contribuciones a las Ciencias Sociales, n. 72, p. 3, 2020.

SILVA, Fabiana Ferreira; RIBEIRO, Ana Regina Bezerra; SILVA, Washington Ferreira. Consultoría Organizacional como um Instrumento de Inovação em arranjos produtivos locais. Revista Organizações em Contexto, v. 14, n. 27, p. 29-52, 2018.

VILLA, Patricia et al. O monólogo contábil: uma análise do uso da contabilidade gerencial em micro e pequenas empresas à luz do processo comunicacional segundo Bakhtin". Monografia para obtenção de título de mestrado em contabilidade. UFPR, 2012.

XAVIER, Leonardo Montes; CARRARO, Wendy Beatriz Witt Haddad; RODRIGUES, Ana Tércia Lopes. Indústria 4.0 e avanços tecnológicos da área contábil: perfil, percepções e expectativas dos profissionais. Contexto. Porto Alegre, RS. Vol. 20, n. 45 (maio/ago. 2020), p. 34-50, 2020.

Déposée: Juillet 2021.

Approuvé : Juillet 2021.

RC: 93886

Disponível em: https://www.nucleodoconhecimento.com.br/administration-des- 